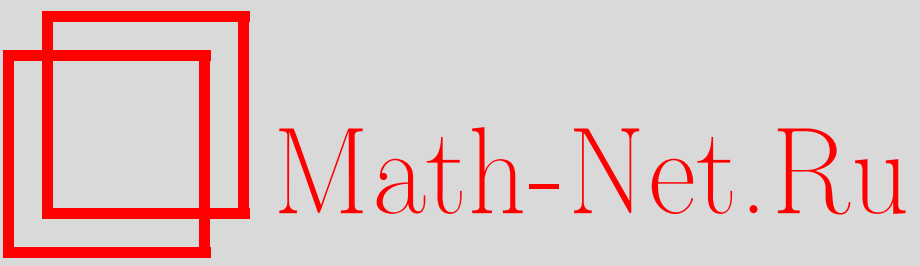

А. В. Глушак, Семейство операторных функций Бесселя, Итоги науки и техн. Сер. Соврем. мат. и ее прил. Темат. обз., 2020, том 187, 36-43

DOI: https://doi.org/10.36535/0233-6723-2020-187-36-43

Использование Общероссийского математического портала Math-Net.Ru подразумевает, что вы прочитали и согласны с пользовательским соглашением

http://www.mathnet.ru/rus/agreement

Параметры загрузки:

IP : 54.224 .60 .19

26 апреля 2023 г., 14:26:30 


\title{
СЕМЕЙСТВО ОПЕРАТОРНЫХ ФУНКЦИЙ БЕССЕЛЯ
}

\author{
(c) 2020 г. $\quad$ А. В. ГЛУШАК
}

\begin{abstract}
АннотАция. Введены в рассмотрение семейство операторных функций Бесселя и генератор этого семейства. Исследованы их свойства, установлен критерий равномерной корректности задачи Коши для уравнение Эйлера-Пуассона-Дарбу и указаны связи этого семейства с рядом других разрешающих операторов.
\end{abstract}

Ключевые слова: операторная функция Бесселя, генератор, уравнение Эйлера-ПуассонаДарбу, задача Коши, критерий равномерной корректности.

\section{FAMILY OF BESSEL OPERATOR FUNCTIONS}

\author{
(c) 2020 A. V. GLUSHAK
}

\begin{abstract}
A family of Bessel operator functions and a generator of this family are introduced and considered. Their properties are studied, a criterion of the uniform well-posedness of the Cauchy problem for the Euler-Poisson-Darboux equation is obtained, and the connections of this family with a series of other solving operators are found.
\end{abstract}

Keywords and phrases: Bessel operator function, generator, Euler-Poisson-Darboux equation, Cauchy problem, uniform well-posedness criterion.

AMS Subject Classification: $34 \mathrm{G} 10$

1. Введение. Исследование дифференциальных уравнений с неограниченными операторными коэффициентами, действующими в банаховом пространстве $E$, стимулирует развитие теории разрешающих операторов соответствующих начальных задач. В результате исследований эволюционных уравнений первого порядка

$$
u^{\prime}(t)=A u(t)
$$

возникли полугруппы линейных операторов $T(t)$, а при изучении уравнения второго порядка (абстрактного волнового уравнения)

$$
u^{\prime \prime}(t)=A u(t)
$$

- операторные косинус-функции $C(t)$. Ослабление требований на разрешающие операторы задачи Коши для абстрактных дифференциальных уравнений первого и второго порядков привело к понятию проинтегрированной полугруппы и проинтегрированной операторной косинус-функции. Терминологию и литературные источники см. в $[13,14]$ и обзорных работах $[1,17]$.

В дальнейшем в работах $[3,6,8,9,12]$ были введены в рассмотрение операторные функции Бесселя, Струве и Лежандра как разрешающие операторы при исследовании соответственно уравнений Эйлера-Пуассона-Дарбу (ЭПД), Бесселя-Струве и Лежандра.

В настоящей работе излагается другой подход к построению семейства операторных функций Бесселя. Так же, как и в теории полугрупп и операторных косинус-функций, семейство операторных функций Бесселя исследуется вначале независимо от дифференциального уравнения, с

Работа выполнена при поддержке Российского фонда фундаментальных исследований (проект № 19-01-00732). 
которым в итоге оно будет связано. Важную роль в построении семейства играет зависящий от параметра $k>0$ оператор обобщенного сдвига $T_{s}^{t}$, определяемый равенством (см. [16])

$$
T_{s}^{t} Y(s)=\frac{\Gamma(k / 2+1 / 2)}{\Gamma(1 / 2) \Gamma(k / 2)} \int_{0}^{\pi} Y\left(\sqrt{s^{2}+t^{2}-2 s t \cos \varphi}\right) \sin ^{k-1} \varphi d \varphi,
$$

где $\Gamma(\cdot)$ - гамма-функция Эйлера $s, t \geqslant 0$. Оператор обобщенного сдвига зависит от параметра $k>0$, но, следуя [16], этот факт в его записи отмечать не будем.

Укажем также, что в настоящей работе мы обходимся понятием интеграла от непрерывной функции, но в случае необходимости можно использовать интеграл Бохнера от функции со значением в банаховом пространстве.

2. Семейство операторных функций Бесселя. Пусть $k>0$ и $Y_{k}(\cdot):[0, \infty) \rightarrow B(E)-$ операторная функция, действующая в пространство линейных ограниченных операторов $B(E)$.

Определение 1. Зависящее от параметра $k>0$ сильно непрерывное семейство линейных ограниченных операторов $Y_{k}(t):[0, \infty) \rightarrow B(E)$ называется операторной функиией Бесселя (ОФБ) если выполнены следующие условия:

(a) $Y_{k}(0)=I$

(b) $Y_{k}(t) Y_{k}(s)=T_{s}^{t} Y_{k}(s), s, t \geqslant 0$;

(c) существуют такие $M \geqslant 1, \omega \geqslant 0$, что $\left\|Y_{k}(t)\right\| \leqslant M e^{\omega t}, t \geqslant 0$.

С семейством ОФБ будет тесно связан дифференциальный оператор Бесселя

$$
B_{k}=\frac{d^{2}}{d t^{2}}+\frac{k}{t} \frac{d}{d t}
$$

для которого, в случае необходимости, будем указывать переменную, по которой он действует: $B_{k}=B_{k, t}$. В дальнейшем также используется обозначение $Y_{k}^{\prime}(t) x=\left(Y_{k}(t) x\right)^{\prime}$.

Определение 2. Генератором ОФБ $Y_{k}(t)$ называется оператор $A$ с областью определения $D(A)$, состоящей из тех $x \in E$, для которых функция $Y_{k}(t) x$ дважды дифференцируема в точке $t=0$, и определяемый равенством

$$
A x=\lim _{t \rightarrow 0+} B_{k} Y_{k}(t) x .
$$

Теорема 1. Если оператор $A$ является генератором ОФБ $Y_{k}(t)$, то область определения $D(A)$ плотна в E. Более того, в $E$ плотно множество элементов, на которых определенъ все степени оператора $A$.

Доказательство. Пусть $V$ - множество числовых функций $v(t)$, гладких и финитных на $(0, \infty)$. Рассмотрим множество $E(V) \subset E$ элементов вида

$$
y=\int_{0}^{\infty} v(s) Y_{k}(s) x d s, \quad x \in E,
$$

и покажем, что $y \in D(A)$. Учитывая условие b) определения 1 и свойства оператора преобразования $T_{s}^{t}$, для рассматриваемых $y$, после интегрирования по частям, получим

$$
\begin{aligned}
B_{k, t} Y_{k}(t) y=\int_{0}^{\infty} v(s) B_{k, t} T_{s}^{t} Y_{k}(s) x d s=\int_{0}^{\infty} v(s) B_{k, s} T_{s}^{t} Y_{k}(s) x d s= \\
\quad=\int_{0}^{\infty}\left(v^{\prime \prime}(s)-k\left(\frac{v(s)}{s}\right)^{\prime}\right) T_{s}^{t} Y_{k}(s) x d s,
\end{aligned}
$$


откуда, переходя к пределу при $t \rightarrow 0+$, получаем, что $y \in D(A)$ и

$$
A y=\int_{0}^{\infty}\left(v^{\prime \prime}(s)-k\left(\frac{v(s)}{s}\right)^{\prime}\right) Y_{k}(s) x d s .
$$

Покажем теперь, что $E(V)$ плотно в $E$. Если бы это было не так, то существовал бы линейный ограниченный функционал $f \neq 0, f \in E^{*}$ такой, что $f(E(V))=0$. Тогда

$$
\int_{0}^{\infty} v(s) f\left(Y_{k}(s) x\right) d s=0, \quad x \in E,
$$

и поэтому, в силу произвольности $v(s) \in V, f\left(Y_{k}(s) x\right) \equiv 0$ при $s>0$. Отсюда в пределе при $s \rightarrow 0$ получим $f(x)=0$ для любого $x \in E$, и поэтому $f=0$, что приводит к противоречию. Следовательно, $E(V)$ плотно в $E$. Поскольку $E(V) \subset D(A)$, то и $D(A)$ плотно в $E$.

Аналогично устанавливается, что в $E$ плотно множество элементов, на которых определены все степени оператора $A$. Теорема доказана.

Теорема 2. Пусть $Y_{k}(t)$ - операторная функиия Бесселя и $A$-ее генератор. Тогда для любых $t, s \geqslant 0 u x \in D(A)$ справедливы равенства

$$
\begin{aligned}
Y_{k}(t) Y_{k}(s) & =Y_{k}(s) Y_{k}(t), \\
A Y_{k}(t) x & =Y_{k}(t) A x .
\end{aligned}
$$

Доказательство. Равенство (1) следует из определения семейства ОФБ и свойств оператора обобщенного сдвига $T_{s}^{t}$. Покажем далее, что

$$
B_{k, s} Y_{k}(s) Y_{k}(t) x=Y_{k}(t) B_{k, s} Y_{k}(s) x, \quad x \in D(A) .
$$

Действительно, в силу теоремы 1 и характеристического свойства оператора обобщенного сдвига (см. [13])

$$
\lim _{h \rightarrow 0+} \frac{T_{s}^{h} Y_{k}(s) x-Y_{k}(s) x}{h^{2}}=\frac{1}{2(k+1)} B_{k, s} Y_{k}(s) x
$$

будем иметь

$$
\begin{aligned}
& B_{k, s} Y_{k}(s) Y_{k}(t) x=2(k+1) \lim _{h \rightarrow 0+} \frac{T_{s}^{h} Y_{k}(s) Y_{k}(t) x-Y_{k}(s) Y_{k}(t) x}{h^{2}}= \\
& \begin{array}{r}
=2(k+1) \lim _{h \rightarrow 0+} \frac{Y_{k}(t)\left(Y_{k}(h) Y_{k}(s) x-Y_{k}(s) x\right)}{h^{2}}=2(k+1) Y_{k}(t) \lim _{h \rightarrow 0+} \frac{T_{s}^{h} Y_{k}(s) x-Y_{k}(s) x}{h^{2}}= \\
=Y_{k}(t) B_{k, s} Y_{k}(s) x,
\end{array}
\end{aligned}
$$

и тем самым равенство (3) установлено.

Переходя в (3) к пределу при $s \rightarrow 0+$, получим равенство (2). Теорема доказана.

Теорема 3. Пусть $Y_{k}(t)$ - операторная функиия Бесселя и $A$-ее генератор. Если $x \in D(A)$ $u t>0, \operatorname{mo} Y_{k}(t) x \in D(A) u$

$$
A Y_{k}(t) x=B_{k, t} Y_{k}(t) x
$$

Доказательство. Поскольку при $s>0$ справедливы равенства

$$
B_{k, s} Y_{k}(s) Y_{k}(t) x=B_{k, s} T_{t}^{s} Y_{k}(t) x=B_{k, t} T_{t}^{s} Y_{k}(t) x=B_{k, t} Y_{k}(s) Y_{k}(t) x=Y_{k}(s) B_{k, t} Y_{k}(t) x,
$$

то, переходя к пределу при $s \rightarrow 0+$, получим требуемые утверждения. Теорема доказана. 
3. Задача Коши для уравнения Эйлера-Пуассона-Дарбу. Обратимся теперь к задаче Коши, с которой связана ОФБ $Y_{k}(t)$.

Определение 3. Решением линейного дифференциального уравнения второго порядка с операторным коэффициентом $A$ называется функция $u(t)$, которая при $t \geqslant 0$ дважды непрерывно дифференцируема, при $t>0$ принимает значения, принадлежащие $D(A)$, т.е., $u(t) \in$ $C^{2}\left(\bar{R}_{+}, E\right) \cap C\left(R_{+}, D(A)\right)$, и удовлетворяет этому уравнению.

Теорема 4. Пусть $Y_{k}(t)$ - операторная функиия Бесселя, $A$-ее генератор и $u_{0} \in D(A)$. Тогда функиия $Y_{k}(t) u_{0}$ является решением уравнения ЭПД

$$
B_{k} u(t) \equiv u^{\prime \prime}(t)+\frac{k}{t} u^{\prime}(t)=A u(t), \quad t>0,
$$

и удовлетворлет начальным условиям

$$
u(0)=u_{0}, \quad u^{\prime}(0)=0 .
$$

Доказательство. Если $u_{0} \in D(A)$, то в силу равенства (4), установленного в теореме 3 , функция $Y_{k}(t) u_{0}$ является решением уравнения (5) и, очевидно, удовлетворяет первому начальному условию в (6). Чтобы проверить, что она удовлетворяет и второму условию в (6), следует уравнение (5) записать в виде

$$
\left(t^{k} u^{\prime}(t)\right)^{\prime}=t^{k} A u(t)
$$

После интегрирования (7) для производной функции $Y_{k}(t) u_{0}$ получим представление

$$
Y_{k}^{\prime}(t) u_{0}=t^{-k} \int_{0}^{t} s^{k} Y_{k}(s) A u_{0} d s,
$$

из которого и следует требуемое утверждение.

Теорема 5. Если $Y_{k}(t)$-операторнал функиия Бесселя, то ее генератор А замкнут.

Доказательство. Пусть $x_{n} \in D(A)$ и $x_{n} \rightarrow x_{0}, A x_{n} \rightarrow y_{0}$. Тогда, в силу теорем 2 и 4 справедливы равенства

$$
\lim _{t \rightarrow 0+} B_{k, t} Y_{k}(t) x_{0}=\lim _{t \rightarrow 0+} \lim _{n \rightarrow \infty} B_{k, t} Y_{k}(t) x_{n}=\lim _{t \rightarrow 0+} \lim _{n \rightarrow \infty} A Y_{k}(t) x_{n}=\lim _{t \rightarrow 0+} Y_{k}(t) y_{0}=y_{0}
$$

Поэтому $x_{0} \in D(A)$ и $A x_{0}=y_{0}$. Теорема доказана.

Определение 4. Задача Коши (5), (6) называется равномерно корректной, если для любого $u_{0} \in D(A)$ существуют заданная на $E$ коммутирующая с $A$ операторная функция $\widetilde{Y}_{k}(t)$ (разрешающий оператор) и числа $M \geqslant 1, \omega \geqslant 0$, такие, что для любого $u_{0} \in D(A)$ функция $u(t)=\widetilde{Y}_{k}(t) u_{0}$ является ее единственным решением и при этом справедливы оценки

$$
\begin{aligned}
\left\|\widetilde{Y}_{k}(t)\right\| & \leqslant M \exp (\omega t), \\
\left\|\widetilde{Y}_{k}^{\prime}(t) u_{0}\right\| & \leqslant M t \exp (\omega t)\left\|A u_{0}\right\| .
\end{aligned}
$$

Множество операторов $A$, для которых задача (5), (6) равномерно корректна, обозначим через $G_{k}$, при этом $G_{0}$ - множество генераторов операторной косинус-функции и $C(t)=Y_{0}(t)$.

Укажем, что случай абстрактного волнового уравнения $(k=0$ в $(5))$ подробно рассмотрен в $[20,21,23]$. В этих работах установлено, что задача (5), (6) при $k=0$ равномерно корректна только тогда, когда оператор $A$ является генератором операторной косинус-функции $C(t)$. В этих же работах приводятся необходимые и достаточные условия того, что оператор $A$ является генератором операторной косинус-функции, которые формулируются в терминах оценки нормы резольвенты $R(\lambda)=(\lambda I-A)^{-1}$ оператора $A$ и ее производных.

Пусть задача Коши (5), (6) равномерно корректна, т.е., $A \in G_{k}$. Введем в рассмотрение разрешающий оператор $\widetilde{Y}_{k}(t)$, ставящий в соответствие элементу $u_{0} \in D(A)$ значение решения $u(t)$ этой задачи в момент $t>0$. В силу определения 4 , этот оператор линейный непрерывный и может 
быть по непрерывности расширен до линейного ограниченного оператора, определенного на всем банаховом пространстве $E$. В дальнейшем будет установлено, что разрешающий оператор $\widetilde{Y}_{k}(t)$ представляет собой ОФБ, т.е., удовлетворяет условиям (а)-(c) определения 1 , а оператор $A-$ ее генератор.

Подробное доказательство формулируемых далее теорем 6-12 приведено в [12].

Теорема 6. Если задача (5), (6) равномерно корректна $u \operatorname{Re} \lambda>\omega$, то $\lambda^{2}$ принадлежит регулярному множеству $\rho(A)$ и для любого $x \in E$ справедливо представление

$$
\lambda^{(1-k) / 2} R\left(\lambda^{2}\right) x=\frac{2^{(1-k) / 2}}{\Gamma(k / 2+1 / 2)} \int_{0}^{\infty} K_{\nu}(\lambda t) t^{(k+1) / 2} \widetilde{Y}_{k}(t) x d t
$$

где $K_{\nu}(\cdot)$ - функиия Макдональда или модифичированная функиия Бесселя третъего рода порядка $\nu=(k-1) / 2$.

Таким образом, спектр $\sigma(A)$ оператора $A \in G_{k}$ всегда лежит левее некоторой параболы.

Теорема 7. Пусть задача (5), (6) равномерно корректна и пусть $\widetilde{Y}_{k}(t)$ - разрешающий оператор для этой задачи. Тогда оператор $A$ является генератором $C_{0}$-полугруппы $T(t)$, и для этой полугруппы справедливо представление

$$
T(t) x=\frac{1}{2^{k} \Gamma(k / 2+1 / 2) t^{k / 2+1 / 2}} \int_{0}^{\infty} s^{k} \exp \left(-s^{2} /(4 t)\right) \widetilde{Y}_{k}(s) x d s, \quad x \in E .
$$

Из представления (11) следует, что полугруппу $T(t)$ можно продолжить до операторной функции, аналитической в некотором секторе, поэтому при нахождении критерия равномерной корректности задачи (5), (6) можно ограничиться классом операторов, которые являются генераторами аналитических $C_{0}$-полугрупп $T(t)$. Обозначим этот класс операторов через $G$. Критерии того, что $A \in G$ могут быть найдены в обзорной работе [1].

В работе [22] показано, что если $A \in G$, то при $\operatorname{Re} \lambda>\omega$ для $\alpha>0$ существует дробная степень резольвенты $R(\lambda)$, которая имеет вид

$$
R^{\alpha}(\lambda) x=\frac{1}{\Gamma(\alpha)} \int_{0}^{\infty} t^{\alpha-1} \exp (-\lambda t) T(t) x d t, \quad x \in E .
$$

Помимо установленного в теореме 6, сформулируем далее еще одно необходимое условие равномерной корректности задачи (5), (6).

Теорема 8. Если задача (5), (6) равномерно корректна $u \operatorname{Re} \lambda>\omega$, то $\lambda^{2}$ принадлежит резольвентному множеству $\rho(A)$ оператора $A$, для дробной степени резольвенты справедливо представление

$$
R^{1+k / 2}\left(\lambda^{2}\right)=\frac{1}{\Gamma(k+1) \lambda} \int_{0}^{\infty} t^{k} \exp (-\lambda t) \widetilde{Y}_{k}(t) d t
$$

и при этом выполняются ощенки

$$
\left\|\frac{d^{n}}{d \lambda^{n}}\left(\lambda R^{1+k / 2}\left(\lambda^{2}\right)\right)\right\| \leqslant \frac{M \Gamma(k+n+1)}{(\operatorname{Re} \lambda-\omega)^{k+n+1}}, \quad n=0,1,2, \ldots
$$

В действительности, оценки (13) будут являться и достаточным условием равномерной корректности задачи (5), (6).

Теорема 9. Пусть $A \in G$, выполнены оценки (13) и $F_{k}(\lambda)=\Gamma(k+1) \lambda R^{1+k / 2}\left(\lambda^{2}\right)$. Тогда задача (5), (6) равномерно корректна, и при этом разрешающий оператор $\widetilde{Y}_{k}(t)$ для этой задачи определяется равенством

$$
\widetilde{Y}_{k}(t)=\lim _{n \rightarrow \infty} e^{-n t}\left(I+\sum_{m=0}^{\infty}(-1)^{m} \frac{n^{k+2 m+2}}{m ! \Gamma(k+m+2)} t^{m+1} F_{k}^{(m)}(n)\right) .
$$


В частности, на элементах из области определения оператора $A$ он имеет вид

$$
\widetilde{Y}_{k}(t) u_{0}=\frac{\Gamma(k+1)}{2 \pi i t^{k}} \int_{\sigma-i \infty}^{\sigma+i \infty} e^{\lambda t} \lambda R^{1+k / 2}\left(\lambda^{2}\right) u_{0} d \lambda, \quad u_{0} \in D(A), \quad \sigma>\omega .
$$

Теоремы 8 и 9 объединяются в следующий критерий.

Теорема 10 (критерий равномерной корректности). Пусть оператор $A$ является генератором аналитической $C_{0}$-полугруппь. Для того чтобы задача (5), (6) была равномерно корректной, необходимо и достаточно, чтобы при некоторых постоянных $M \geqslant 1, \omega \geqslant 0$ число $\lambda^{2} c$ $\operatorname{Re} \lambda>\omega$ принадлежало резольвентному множеству оператора $A$ и для дробной степени резольвенты оператора А были выполнены оценки (13).

Теорема 11. Пусть задача Коши (5), (6) равномерно корректна и $\widetilde{Y}_{k}(t)$ - разрешающий оператор для этой задачи. Тогда $\widetilde{Y}_{k}(t)$ удовлетворяет условиям (а)-(c) определения 1 и, следовательно, является ОФБ, т.е., $\widetilde{Y}_{k}(t)=Y_{k}(t)$.

Итак, если при изучении задачи Коши (5), (6) ограничиться только замкнутым оператором $A$ с плотной областью определения, то класс уравнений, для которых эта задача равномерно корректна, совпадает с классом уравнений, у которых оператор $A$ является генератором ОФБ $Y_{k}(t)$, а сама ОФБ при этом является разрешающим оператором $\widetilde{Y}_{k}(t)=Y_{k}(t)$ для рассматриваемой задачи. Примеры ОФБ и соответствующих генераторов приводятся в [12].

Семейство ОФБ $Y_{k}(t)$ зависит от параметра $k>0$. Указанная зависимость содержится в приводимой далее формуле сдвига по этому параметру.

Теорема 12. Пусть $0 \leqslant m<k u A \in G_{m}$ - генератор ОФБ $Y_{m}(t)$. Тогда $A \in G_{k} \supset G_{m}, n p u$ этом соответствующая ОФБ $Y_{k}(t)$ имеет вид

$$
Y_{k}(t)=\frac{2 \Gamma(k / 2+1 / 2)}{\Gamma(m / 2+1 / 2) \Gamma(k / 2-m / 2)} \int_{0}^{1} s^{m}\left(1-s^{2}\right)^{(k-m) / 2-1} Y_{m}(t s) d s .
$$

В частности, если $A \in G_{0} \subset G_{k}$ является генератором КОФ $C(t)$, то

$$
Y_{k}(t)=\frac{2 \Gamma(k / 2+1 / 2)}{\Gamma(1 / 2) \Gamma(k / 2)} \int_{0}^{1}\left(1-s^{2}\right)^{k / 2-1} C(t s) d s, \quad k>0,
$$

при этом (см. [4]) равномерно по $t \in\left[0, t_{0}\right], t_{0}>0$

$$
\lim _{k \rightarrow 0} Y_{k}(t) x=C(t) x, \quad x \in E .
$$

Ранее в работах $[11,18]$ именно равенство $(15)$ было использовано при построении ОФБ, интерпретируемой как разрешающий оператор для рассматриваемых в этих работах дифференциальных уравнений. Дальнейшее обобщение формулы сдвига по параметру приводится в [10].

Из равенства (14) вытекают следующие формулы для производных ОФБ:

$$
Y_{k}^{\prime}(t) u_{0}=\frac{t}{k+1} Y_{k+2}(t) A u_{0}, \quad \lim _{t \rightarrow 0} Y_{k}^{\prime \prime}(t) u_{0}=\frac{1}{k+1} A u_{0}, \quad u_{0} \in D(A) .
$$

Завершая этот раздел, отметим, что случай возмущения оператора $A \in G_{k}$ ограниченным оператором исследован в [2], а если $A \in G_{k}$ и $B \in G_{m}, m<k$, то вопрос о принадлежности оператора $A+B$ некоторому классу корректности рассмотрен в [5].

4. Заключение. В этом разделе мы укажем на связи ОФБ $Y_{k}(t)$ с рядом других разрешающих операторов.

При $k<0$ и $A \in G_{2-k}$ операторная функция

$$
Z_{k}(t)=\frac{t^{1-k}}{1-k} Y_{2-k}(t)
$$


определяет решение уравнения ЭПД (5), удовлетворяющее условиям

$$
u(0)=0, \quad \lim _{t \rightarrow 0+} t^{k} u^{\prime}(t)=u_{1},
$$

и поэтому она названа операторной функцией Бесселя с отрицательным индексом. В работе [9] доказано, что множество $G_{2-k}$ является классом корректности весовой задачи (5), (16), а функция $Z_{k}(t) u_{1}, u_{1} \in D(A)$ - ее единственное решение.

Отметим, что при $k<0$ задача Коши для уравнения ЭПД (5) с условиями

$$
u(0)=0, \quad u^{\prime}(0)=u_{1}
$$

не является корректной ввиду потери единственности (см. [19]).

В [8] при $k>0$ введена в рассмотрение операторная функция Струве $L_{k}(t)$, которая связана с ОФБ $Y_{k}(t)$ формулой

$$
L_{k}(t) x=\frac{\sqrt{\pi} \Gamma(k / 2+1)}{\Gamma(k / 2+1 / 2)} \int_{0}^{t}{ }_{2} F_{1}\left(\frac{1}{2}, \frac{k}{2} ; 1 ; 1-\frac{t^{2}}{\tau^{2}}\right) Y_{k}(\tau) x d \tau, \quad A \in G_{k}, \quad x \in E,
$$

где ${ }_{2} F_{1}(a, b ; c ; z)$ - гипергеометрическая функция Гаусса.

Если $k>0, A \in G_{k}, u_{0}, u_{1} \in D(A)$, то функция $u(t)=Y_{k}(t) u_{0}+L_{k}(t) u_{1}$ будет единственным решением уравнения Бесселя-Струве

$$
u^{\prime \prime}(t)+\frac{k}{t}\left(u^{\prime}(t)-u^{\prime}(0)\right)=A u(t), \quad t>0,
$$

удовлетворяющим условиям

$$
u(0)=u_{0}, \quad u^{\prime}(0)=u_{1} .
$$

Важно отметить, что наличие в уравнении (17) заданной при $t=0$ нагрузки $u^{\prime}(0)$ меняет постановку начальной задачи при $k>0$. Вместо корректной для уравнения ЭПД (5) весовой задачи с условием (16), для уравнения Бесселя-Струве (17) следует рассматривать обычную задачу Коши с условием (18).

Укажем далее на связь ОФБ $Y_{k}(t)$ с разрешающим оператором задачи Коши для уравнения Лежандра

$$
u^{\prime \prime}(t)+k \operatorname{cth} t u^{\prime}(t)+(k / 2)^{2} u(t)=A u(t), \quad t>0, \quad k>0 .
$$

Дифференциальный оператор в левой части уравнения (19) возникает при решении уравнения Лапласа в координатах вытянутого эллипсоида вращения. Как следует из результатов работы [6], корректная постановка начальных условий для абстрактного уравнения Лежандра (19), так же как и для уравнения ЭПД (5), состоит в задании в точке $t=0$ начальных условий

$$
u(0)=u_{0}, \quad u^{\prime}(0)=0 .
$$

В [6] также доказано, что множество операторов $A$, с которыми задача (19), (20) равномерно корректна, совпадает с множеством $G_{k}$, и приведены формулы, связывающие разрешающий оператор этой задачи с ОФБ $Y_{k}(t)$.

Укажем, наконец, на формулу связи ОФБ $Y_{k}(t)$ с проинтегрированной операторной косинусфункцией (ПКОФ), которая установлена в [7]. Пусть $k=2 \alpha>0$ и оператор $A$ является генератором $\alpha$ раз ПКОФ $C_{\alpha}(t), u_{0} \in D(A)$. Тогда задача $(5),(6)$ равномерно корректна, т.е. $A \in G_{k}$, и соответствующая ОФБ представима в виде

$$
Y_{k}(t) u_{0}=\frac{2^{\alpha} \Gamma(\alpha+1 / 2)}{\sqrt{\pi} t^{\alpha}}\left(C_{\alpha}(t) u_{0}-\int_{0}^{1} P_{\alpha-1}^{\prime}(\tau) C_{\alpha}(t \tau) u_{0} d \tau\right),
$$

где $P_{\nu}(t)$ - сферическая функция Лежандра (см. [15, с. 205]). 


\section{СПИСОК ЛИТЕРАТУРЫ}

1. Васильев В. В., Крейн С. Г., Пискарев С. И. Полугруппы операторов, косинус-оператор функции и линейные дифференциальные уравнения// Итоги науки и техн. Мат. анализ. $-1990 .-28 .-$ С. $87-$ 202.

2. Глушак А. В. О возмущении абстрактного уравнения Эйлера-Пуассона-Дарбу// Мат. заметки. 1996. - 60, № 3. - С. 363-369.

3. Глушак А. В. Операторная функция Бесселя// Докл. РАН. - 1997. - 352, № 5. - С. 587-589.

4. Глушак A. В. Регулярное и сингулярное возмущения абстрактного уравнения Эйлера-ПуассонаДарбу// Мат. заметки. - 1999. - 66, № 3. - С. 364-371.

5. Глушак A. В. Операторная функция Бесселя и связанные с нею полугруппы и модифицированное преобразование Гильберта// Диффер. уравн. - 1999. - 35, № 1. - С. 128-130.

6. Глушак A. В. Операторная функция Лежандра// Изв. РАН. Сер. мат. - 2001. - 65, № 6. - С. 3-14.

7. Глушак $A$. В. О связи проинтегрированной косинус-оператор-функции с операторной функцией Бесселя// Диффер. уравн. - 2006. - 42, № 5. - С. 583-589.

8. Глушак A. В. Абстрактная задача Коши для уравнения Бесселя-Струве// Диффер. уравн. - 2017. - 53, № 7. - С. 891-905.

9. Глушак А. В. Критерий разрешимости весовой задачи Коши для абстрактного уравнения ЭйлераПуассона-Дарбу// Диффер. уравн. - 2018. - 54, № 5. - С. 627-637.

10. Глушак A. В. Операторная формула сдвига решения задачи Коши для абстрактного уравнения Эйлера-Пуассона-Дарбу// Мат. заметки. - 2019. - 105, № 5. - С. 656-665.

11. Глушак А. В., Кононенко В. И., Шмулевич С. Д. Об одной сингулярной абстрактной задаче Коши// Изв. вузов. Мат. - 1986. - № 6. - С. 55-56.

12. Глушак A. В., Покручин O. А. Критерий разрешимости задачи Коши для абстрактного уравнения Эйлера-Пуассона-Дарбу// Диффер. уравн. - 2016. - 52, № 1. - С. 41-59.

13. Голдстейн Дж. Полугруппы линейных операторов и их приложения. - Киев: Вища школа, 1989.

14. Крейн С. Г. Линейные дифференциальные уравнения в банаховом пространстве. - М.: Наука, 1967.

15. Лебедев Н. Н. Специальные функции и их приложения. - М.: Физматгиз, 1963.

16. Левитан Б. М. Разложение по функциям Бесселя в ряды и интегралы Фурье// Усп. мат. наук. 1951. - 1, № 2 (42). - C. 102-143.

17. Мельникова И. В., Филинков А. И. Интегрированные полугруппы и $C$-полугруппы. Корректность и регуляризация дифференциально-операторных задач// Усп. мат. наук. -1994 . -49 , № 6 (300). C. $111-150$.

18. Орлов В. П. О слабо вырождающихся гиперболических уравнениях// Диффер. уравн. - 2003. - 39, № 10. - C. 1409-1419.

19. Bresters D. W. On the Euler-Poisson-Darboux equation// SIAM J. Math. Anal. — 1973. - 4, № 1. P. 31-41.

20. Da Prato G., Giusti E. Una caratterizzazione dei generatori di funzioni coseno astratte// Boll. Union. Mat. Ital. - 1967. - 22. - P. 357-362.

21. Fattorini H. O. Ordinary differential equations in linear topological space, II// J. Differ. Equ. - 1969. 6. - P. 50-70.

22. Fattorini H. O. A note on fractional derivatives of semigroups and cosine functions// Pac. J. Math. - 1983. - 109, № 2. - P. 335-347.

23. Sova M. Cosine operator functions// Rozpr. mat. - 1966. - 49. - P. 1-47.

Глушак Александр Васильевич

Белгородский государственный национальный исследовательский университет

E-mail: aleglu@mail.ru, Glushak@bsu.edu.ru 